\title{
ESTIMATION OF THE NUMBER OF EXCESS CLAIMS BY MEANS OF THE CREDIBILITY THEORY
}

\author{
E. Straub \\ Zurich
}

\section{Preliminary Remarks}

The purpose of this paper is to describe a possible application on the rating of excess of loss covers of Mr. Bühlmann's work on Experience Rating and Credibility 1) ${ }^{2}$ ). One of the most important problems in connection with the rating of such treaties consists in estimating the number of excess claims and the average excess claim amount. Especially with cases where claims data are scarce there is a temptation to estimate these two quantities by means of the credibility theory. This approach leads, on the one hand, to a relatively complicated formula when considering the average excess claim amount, and, on the other, to a rather simple one for the credibility factor of the number of excess claims.

\section{Notations. Description of Model}

We consider a portfolio consisting of $N$ excess of loss treaties $i=\mathrm{I}, 2, \ldots N$

$n_{i}$ number of risks under observation

$\left.\begin{array}{l}R_{i} \text { first risk (retention) } \\ k_{i} \text { observed number of claims exceeding } R_{i}\end{array}\right\}$ for treaty $i$

For treaty $i$, let $k_{i}^{*}$ (stochastic variable) be the number of all claims (not only excess claims) per year.

1) Optimale Prämienstufensysteme, Mitteilung der Vereinigung schweizerischer Versicherungsmathematiker, 64. Band, Heft 2.

2) Experience Rating and Credibility, The ASTIN-Bulletin, Vol. IV, Part III. 
We assume that each of the variables $k_{i}{ }^{*}$ is Poisson-distributed or compound Poisson ${ }^{3}$ ) distributed, respectively

$$
\operatorname{Prob}\left[k_{i}^{*}=n\right]=\frac{\left(n_{i} \lambda\right)^{n}}{n !} e^{-n_{i} \lambda}
$$

or

$$
\operatorname{Prob}\left[k_{i}^{*}=n\right]=\int \frac{\left(n_{i} \vartheta\right)^{n}}{n !} e^{-n_{i} \vartheta d G(\vartheta)}
$$

where $G(9)$ is an arbitrary distribution with

Thus we have

$$
\int \vartheta d G(\vartheta)=\lambda
$$

$$
E\left[k_{i}^{*}\right]=n_{i} \lambda
$$

From this assumption on the distribution of all claims it follows that the number of excess claims above any retention $R$ is again Poisson-distributed or compound Poisson-distributed. This property is easily verified below in the case of the normal Poisson law*).

For any of the above $N$ treaties let $k^{*}$ be the number of all claims, $k$ the number of those exceeding a given first risk $R$ and $V(x)$ the distribution of the amount of one claim.

With this notation we get

$$
\operatorname{Prob}\left[k=m \mid k^{*}=n\right]=\left(\begin{array}{l}
n \\
m
\end{array}\right) V^{n-m}(R)[\mathrm{I}-V(R)]^{m}
$$

Under the assumption that

$$
\operatorname{Prob}\left[k^{*}=n\right]=\frac{a}{n !} e^{-a}
$$

we have

$$
P_{m}(R)=\operatorname{Prob}[k=m]=\sum_{n=0}^{\infty} \frac{a^{n}}{n} e^{-a}\left(\begin{array}{c}
n \\
m
\end{array}\right) V^{n-m}(R)[\mathrm{I}-V(R)]^{m}
$$

$\left.{ }^{3}\right)$ Using Philipson's terminology in his article "on the difference between the concepts "compound" and "composed" Poisson processes", ASTINBulletin Vol. II, Part III.

*) For the sake of simplicity the calculations are only made for the simple Poisson-distribution, although it can be proved, without further difficulties that the results of this note hold true for compound Poisson laws as well. 
or

$$
\begin{aligned}
P_{m}(R)= & \frac{a^{m}[\mathrm{I}-V(R)]^{m}}{m !} e^{-a} \sum_{n-0}^{\infty} \frac{a^{n-m} V^{n-m}(R)}{(n-m) !} \\
& =\frac{a^{m}[\mathrm{I}-V(R)]}{m !} e^{-a[1-V(R)]}
\end{aligned}
$$

which means that the number of claims exceeding $R$ is again Poisson-distributed but with the new parameter

$$
a(R)=a H(R), H(R)=I-V(R)
$$

We further assume the amount $x_{i}$ of one claim to be distributed with $V_{i}(x)$

$$
V_{i}(x)=\operatorname{Prob}\left[x_{i} \leqslant x\right] \text { for treaty } i
$$

In accordance with $\mathrm{Mr}$. Bühlmann's notation we denote with $S(\lambda)$ the structural function of the portfolio. $S(\lambda)$ therefore is the distribution of $\lambda$ which is looked upon as stochastic variable.

Further notations: $\mu=\int \lambda d S(\lambda), \tau^{2}=\int(\lambda-\mu)^{2} d S(\lambda)$

Finally we simply write

$$
\int g\left(k_{i}\right) d P\left(k_{i} \mid \lambda\right) \text { for the sum } \sum_{\boldsymbol{x}_{i}=0}^{\infty} g\left(k_{i}\right) \frac{\left[n_{i} H_{i}\left(R_{i}\right) \lambda\right]^{k_{i}}}{k_{i} !} e^{\left.-n_{i} H_{i}\left(R_{i}\right)\right\rangle}
$$

for any arbitrary function $g\left(k_{i}\right) ; H_{i}\left(R_{i}\right)=\mathrm{I}-V_{i}\left(R_{i}\right)$

\section{Calculation of Credibility Factors}

The estimation function for the true parameter has to be chosen so that the average portfolio risk is minimized, i.e.

$$
\begin{gathered}
R\left(\alpha_{1}, \alpha_{2}, \ldots \alpha_{N} ; \beta_{1}, \beta_{2}, \ldots \beta_{N}\right)=R(\alpha, \beta) \\
\sum_{i=1}^{N} \int\left[\alpha_{i} k_{i}+\beta_{i} n_{i} H_{i}\left(R_{i}\right) \mu-n_{i} H_{i}\left(R_{i}\right) \lambda\right]^{2} d P\left(k_{i} \mid \lambda\right) d S(\lambda)=\text { minimum }
\end{gathered}
$$

As can be seen immediately from this condition, we limit our investigation to the special case where

i) the error function is quadratic 
ii) the optimation is only made within the set of all estimation functions which can be written as a linear combination of $k_{i}$ (observation on the individual treaty $i$ ) and $n_{i} H_{i}\left(R_{i}\right) \mu$ (average parameter-value over the whole portfolio).

Integration leads to

$$
\begin{gathered}
R(\alpha, \beta)=\sum_{i=1}^{N}\left[\alpha_{i}^{2} n_{i} H_{i}\left(R_{i}\right) \mu+\beta_{i}^{2} n_{i}^{2} H_{i}^{2}\left(R_{i}\right) \mu^{2}\right. \\
\left.-2 \beta_{i}\left(I-\alpha_{i}\right) n_{i}^{2} H_{i}^{2}\left(R_{i}\right) \mu^{2}+\left(I-\alpha_{i}\right)^{2} n_{i}^{2} H_{i}^{2}\left(R_{i}\right)\left(\mu^{2}+\tau^{2}\right)\right]
\end{gathered}
$$

and from this expression we get by differentiating with respect to $\alpha_{i}$ and $\beta_{i}$

$$
\begin{gathered}
\beta_{i}=\mathrm{I}-\alpha_{i} \\
\alpha_{i}=\frac{n_{i} H_{i}\left(R_{i}\right) \tau^{2}}{\mu+n_{i} H_{i}\left(R_{i}\right) \tau^{2}}
\end{gathered}
$$

or

$$
\alpha_{i}=\frac{n_{i}}{n_{i}+\varkappa_{i}} \quad, \quad \varkappa_{i}=\frac{\mu}{H_{i}\left(R_{i}\right) \tau^{2}}
$$

so that the credibility factor $\alpha_{i}$ is written in the same form as in Mr. Bühlmann's Paper (2), i.e.

$$
\alpha=\frac{n}{n+x} \quad, \quad \alpha=\frac{E\left[\sigma^{2}(\vartheta)\right]}{\operatorname{Var}[\mu(\vartheta)]}
$$

(Putting

$$
\sigma^{2}(\vartheta)=H_{i}\left(R_{i}\right) \vartheta, E\left[\sigma^{2}(\vartheta)\right]=H_{i}\left(R_{i}\right) \mu
$$

and

$$
\mu(\vartheta)=H_{i}\left(R_{i}\right) \vartheta \quad, \quad \operatorname{Var}[\mu(\vartheta)]=H_{i}^{2}\left(R_{i}\right) \tau^{2}
$$

we get again

$$
x=\frac{H_{i}\left(R_{i}\right) \mu}{H_{i}^{2}\left(R_{i}\right) \tau^{2}}=\frac{\mu}{H_{i}\left(R_{i}\right) \tau^{2}}
$$

Within the rating of excess of loss treaties the credibility relation for the number of excess claims is therefore

$$
\alpha_{i} \cdot k_{i}+\left(\mathrm{I}-\alpha_{i}\right) n_{i} H_{i}\left(R_{i}\right) \mu
$$


$k_{i}$ being the number of claims exceeding the retention $R_{i}$ as observed for the individual treaty $i$ and

$n_{i} H_{i}\left(R_{i}\right) \mu$ the average expected number of excess claims for treaty $i$, the average to be taken over the whole excess of loss portfolio.

\section{Final Remarks}

Beside the two fundamental properties of credibility factors, namely

i) $\alpha_{i}$ increases with increasing number $n_{i}$ of risks observed

ii) $\alpha_{i}$ increases with increasing variance $\tau^{2}$

we get-as had to be expected in the special case of an excess of loss portfolio- two further rules for fixing the credibility factors, i.e.

i) $\alpha_{i}$ decreases with increasing retention $R_{i}$

ii) $\alpha_{i}$ also depends on the structure of the distribution $V_{i}(x)$

Finally we should like to point out that, e.g. for a Motor excess of loss portfolio consisting only of treaties from the same country, $V_{i}(x)$ can be put equal to $V(x)$, independent of the individual treaty. The same simplification is, however, unrealistic for a Fire portfolio, since within property branches the structure of $V_{i}(x)$ essentially depends-among other facts, such as composition of the portfolio reinsured by treaty $i$-upon the form of proportional reinsurances (quota shares and/or surplus arrangements) usually protecting the ceding company's account, before the excess of loss cover becomes effective. 